\title{
Effects of Nerve Regeneration Therapy on SFI in Nerve Injured Rats
}

\author{
Youn-Bum Sung ${ }^{1}$, Jung-Ho Lee ${ }^{2 *}$ \\ 1712-714 Dept. Physical Therapy, Daegu Univ., Jillyang-eup, Gyeongsan-si, \\ Gyeongsangbuk-do, Republic of Korea. \\ ${ }_{2}^{2}$ (Corresponding Author) 219-705 Dept. Physical Therapy, Kyungdong Univ., \\ Bongpo-ri, Toseong-myeon, Goseong-gun, Gangwon-do, Republic of Korea. \\ I playeryoon@hanmail.net, ${ }^{2}$ ljhcivapt@naver.com
}

\begin{abstract}
The purpose of this study was to evaluate the effect of nerve regeneration therapy on SFI test after peripheral nerve crush injury in each group as time passes. The rats were randomly assigned to a control group $(C G, n=15)$ that would not to receive any therapeutic intervention after being affected by sciatic nerve damage, a CIMT group $(C E G, n=15)$ that would undergo treadmill exercises utilizing CIMT after being affected by sciatic nerve damage, and an exercise group $(E G, n=15)$ that would only undergo treadmill exercise after being affected by sciatic nerve damage. In addition, each group underwent SFI test at day 1, day 7, and day 14 after receiving treatment. As a result of this study, there was no statistically significant difference in all groups at 7 days, but there was a significant difference at 14 days. The post hoc test showed significant differences among all groups at 14 days. Significant differences were found between $C E G$ and EG, EG and CG. This result shows that exercise after peripheral nerve injury is better, and it is better to apply CIMT and exercise than that. These results suggest that sensory fibers and motor fibers were effectively regenerated and re-innervated thanks to the stimulation of cell healing based on the principle of sensory motor integration therapy to improve functional activities.
\end{abstract}

Keywords: Nerve Regeneration Therapy, Sciatic Functional Index, Rat, Nerve injury,

\section{Introduction}

Peripheral nerves tend to have a very slow therapeutic recovery after damage and often show an incomplete degree of recovery. Peripheral nerve injury reduces the ability to control partial or overall motor nerve control in the area of the hernia and reduces the functional activities and quality of life caused by sensory and motor abilities disabilities[1]. ${ }^{1}$

Since disabilities due to peripheral nervous system damage have negative effects on independent functions necessary to engage in daily life activities, getting appropriate treatment is an important element that will affect the promotion of recovery. Treatment methods for peripheral nerve damage include invasive procedures such as injection therapy and surgical restoration, and non-invasive methods such as electrical stimulation therapy, exercise therapy, hydrotherapy, and medication. An important element of treatment for peripheral nerve damage is the formation of an environment that can maximize neuroplasticity. Decreasing the use of the affected area can cause abnormal movement, decreased muscle strength, stiffness, soft

Article history:

Received (September 25, 2017), Review Result (October 4, 2017), Accepted (December 25, 2017) 
tissue shortening, and pain. A clinically developed treatment to control this learned nonuse is Constraint Induced Movement Therapy (CIMT).

CIMT is defined as a therapy characterized by high-intensity training over a short period of time and an enhancement of the function and use of the affected side by restricting the movement of the unaffected side to better induce movement of the affected side[2]. CIMT has been reported to stimulate the sensory motor cortex in the uninjured cortical circuit and the area adjacent to the damaged area through continuous and repeated functional movement learning, thereby facilitating local terminal firing and reorganization of functional synapses to improve precise and delicate movements[3]. However, as patients may feel burdened by the training, experience psychological stress, and face safety issues, Page et al. (2001) performed a modified CIMT. The modified CIMT was applied to constrain the unaffected side for only the five most active hours of a patient's day[4][5]. The therapeutic effects of the modified CIMT were proven by comparing it to the therapeutic effects of other treatments. Although most studies of CIMT have focused on improving upper limb function in patients with central nervous system damage, a more recent study indicates that gait ability and balance were improved through CIMT thanks to a reported improvement of upper limb function. In addition, Rostami et al. (2016) reported that when CIMT was applied to patients with damage to the median or ulnar nerve, it was effective in treating peripheral nerve injuries. Although many studies echo these reports, CIMT is not widely used as a therapeutic intervention in clinical practice at this time[6].

The purpose of this study was to investigate the effects of treadmill exercises utilizing CIMT as performed by rats, in which crushed sciatic nerves were induced, through SFI in order to solve the ethical problem of being unable to apply CIMT to humans.

\section{Study methods}

\subsection{Subject}

This study was conducted using 45 male Sprague-Dawley rats aged 8 weeks and weighing 250-300g. During the experimental treatment period, the temperature of the laboratory was maintained at $23 \pm 2{ }^{\circ} \mathrm{C}$ and the humidity was maintained at $50 \pm 5 \%$. Four rats were housed per cage in the laboratory and light and dark cycles of 12 hours each per day were applied. The rats were randomly assigned to a control group $(\mathrm{CG}, \mathrm{n}=15)$ that would not to receive any therapeutic intervention after being affected by sciatic nerve damage, a CIMT group (CEG, $\mathrm{n}$ $=15$ ) that would undergo treadmill exercises utilizing CIMT after being affected by sciatic nerve damage, and an exercise group (EG, $n=15)$ that would only undergo treadmill exercise after being affected by sciatic nerve damage. In addition, each group underwent SFI test at day 1 , day 7, and day 14 after receiving treatment. Three days after inducing crushed sciatic nerves in the rats, the first intervention method was implemented immediately after confirming the nerve damage. The rats were treated 5 times per week for 2 weeks with the aforementioned therapeutic intervention methods.

\subsection{Experimental procedure \\ 2.2.1 Induction of Crushed Sciatic Nerves}

After removing the hair, the skin was incised about $2 \mathrm{~cm}$ in length, the sciatic nerves were isolated from the muscles surrounding them, and then the area before where they branch into the shin nerve and peroneal nerve-which is about $7 \mathrm{~cm}$ proximal to the ankle joint-was continuously pressed for 30 seconds using a pair of straight hemostatic forceps. To prevent damage due to the blade of the straight hemostatic forceps during compression, the forceps 
were covered with a soft plastic and disinfected with $70 \%$ alcohol before the crush was induced. To apply nerve damage to a certain area with the same force, a marking was made at a point 5 $\mathrm{mm}$ inward from the end of the hemostatic forceps and the same three-step pressure was applied to all rats.

Disinfection was performed to prevent post-crush infections. The wounds were sutured using animal suture threads. Thereafter, the animals were put into plastic cages - four animals were housed in each plastic cage and were introduced to the feeding room for stabilization.

\subsubsection{Constraint Induced Movement Therapy (CIMT)}

The CIMT intervention method in this study was applied to the unaffected hind leg that was not given a crushed sciatic nerve to prevent the overuse of the unaffected side during treadmill exercises, thereby inducing use of the affected side.

CIMT was applied by wrapping the unaffected hind leg-while maintaining the extension of the knee joint and the ankle joints - with a PET bottle to prevent excessive compression of the local region and wrapping the PET bottle with Kinesio tape to affix the bottle to the leg. Exercise on the treadmill was applied thereafter. The CIMT device was removed immediately after completion of the treadmill exercise to minimize stress.

\subsubsection{Treadmill Exercise Treatment Method}

The exercise intervention in this study was an exercise program applied to all groups except for the control group using a small animal treadmill(JD-A-09 type, JEUNGDO Bio \& Plant Co., Ltd., Korea). In a study conducted earlier by Shepherd and Gollnick (1976), the VO2 max of Sprague-Dawley rats were measured with seven steps of speeds with in the range of 16-67 $\mathrm{m} / \mathrm{min}$ and the researchers reported[7]. Based on the results of the study, to minimize stress and adverse effects from exercise intensity in this study, a low-intensity exercise program was applied with an intervention application time of 30 minutes, a gradient of $0^{\circ}$, and an exercise speed of $8 \mathrm{~m} / \mathrm{min}$.

All groups had a resting period of 3 days after the crushed sciatic nerve was induced; sciatic nerve damage was confirmed thereafter and the exercise program was applied to all groups except for the control group. The intervention was applied 5 times per week for 3 weeks and the exercise was performed during a dark cycle time zone (7:00 p.m.) when the rats were active to maintain their biorhythms and enhance the effects of training. To prevent the rats from ceasing to exercise, a device that would apply an electrical stimulation of 15 volts was installed at the back of the belt of the treadmill, thereby inducing continuous exercise.

\subsection{Result measurement method}

To evaluate the effects of the treadmill exercise using CIMT on motor nerves, the SFI of rats across all groups were measured at day 1, day 7, and day 14 after application of the treatment.

Chinese ink was applied to the soles of the rats, which were then induced to walk through a $50 \mathrm{~cm}$ long, $8 \mathrm{~cm}$ wide, and $10 \mathrm{~cm}$ high dark passage with white paper spread on the floor to obtain footprints. A dark room was crafted at the end of the passage in the direction of progression so that the rat could proceed forward. The rats were allowed to walk twice so that they could acquaint themselves with the direction of the passage and each rat was induced to walk three times repeatedly to ensure that footprints were left. In this case, all external elements that could affect the walking habits of the rats were removed[8]. 
For the footprint analysis of the rats, the distance from the heel to the third toe, the distance between the first and fifth toes, and the distance between the second and fourth toes were measured. Using these measured values allowed the SFI to be calculated. The SFI were near 0 in the case of normal rats and toward -100 as the damage would be recorded as more severe[9].

\subsection{Data analysis}

The results of the experiments obtained in this study were described as mean \pm standard deviation. One-way ANOVAs were conducted for comparison and analysis between groups and between measurement times, and ex post facto tests were assessed using LSD. Statistical processing was performed using the PASW Win. 18.0 package and the level of statistical significance $(\alpha)$ was set to 0.05 .

\section{Results}

The purpose of this study was to evaluate the effect of CIMT exercise on SFI test after peripheral nerve crush injury in each group at 1 day, 7 days, and 14 days. As the result, there was no statistically significant difference in all groups at 7 days $(p>.05)$, but there was a significant difference at 14 days $(\mathrm{p}<.05)$. The post hoc test showed significant differences among all groups at 14 days ( $\mathrm{p}<.05$ ). Significant differences were found between CEG and EG, EG and CG (p<.05)Table 1.

Table 1. Comparison of sciatic functional index in each group

\begin{tabular}{cccccc}
\hline & CEG & EG & CG & F & $p$ \\
\hline 1 day & $-82.49 \pm 5.99$ & $-80.89 \pm 5.73$ & $-81.63 \pm 5.19$ & 1.341 & .533 \\
7 days & $-66.34 \pm 6.91$ & $-68.82 \pm 7.33$ & $-72.03 \pm 4.87$ & 5.127 & .069 \\
14 days & $-32.87 \pm 7.01$ & $-40.11 \pm 8.30$ & $-56.39 \pm 5.77$ & 31.533 & $.000^{*}$ \\
\hline
\end{tabular}

${ }^{*} p<.05$, Mean $\pm \mathrm{SD}:$ mean \pm standard deviation, $\mathrm{CEG}:$ constraint-induced movement therapy \& exercise group, EG : exercise group, CG : control group

\section{Discussion}

The purpose of this study was to investigate the effects of treadmill exercises utilizing CIMT performed by rats with crushed sciatic nerves on nerve SFI recovery over time.

CIMT is a type of therapy that receives attention in the field of nerve rehabilitation. CIMT is a method meant to enhance the performance of the affected side by constraining the unaffected side's movements, thereby inducing the use of the affected side. It was developed by Taub and was based on behavioral neuroscience research. The conventional CIMT is a rehabilitation program that constrains the unaffected side to limit its use for $90 \%$ of the patient's waking time for 2 weeks while engaging them in high-intensity training and task repetition of the affected side, thereby inducing the use of the affected upper limbs[10]. Functional assessments related to peripheral nerve recovery are divided into those for motor nerve recovery and sensory nerve recovery. In relation to motor nerve recovery, SFI, which is a functional assessment for nerve damage in rodents, was known as the most reliable test method, making 
functional assessments regarding regeneration after nerve damage possible. The SFI shows the ratio of insufficient functions when the normal gait is regarded as $0 \%$. An SFI value of 0 indicates normal conditions and an SFI value of -100 indicates complete damage. The SFI obtained from the rats with crushed sciatic nerves per measurement period after the rats performed treadmill exercise using CIMT can be an objective index to evaluate the recovery of motor functions[8].

The purpose of this study was to evaluate the effect of CIMT exercise on SFI test after peripheral nerve crush injury in each group as time passes. As a result of this study, there was no statistically significant difference in all groups at 7 days, but there was a significant difference at 14 days. The post hoc test showed significant differences among all groups at 14 days. Significant differences were found between CEG and EG, EG and CG. This result shows that exercise after peripheral nerve injury is better, and it is better to apply CIMT and exercise than that. These results suggest that sensory fibers and motor fibers were effectively regenerated and re-innervated thanks to the stimulation of cell healing based on the principle of sensory motor integration therapy to improve functional activities.

\section{Acknowledgement}

This work was supported by the National Research Foundation of Korea(NRF) grant funded by the Korea government(MSIT) (No. 2017R1C1B5074613).

\section{References}

[1] H. E. Rosberg, K. S. Carlsson, and L. B. Dahlin. Prospective study of patients with injuries to the hand and forearm: Costs, function, and general health. Scandinavian Journal of Plastic and Reconstructive Surgery and Hand Surgery, Vol. 39, (2005), pp.360-369.

[2] A. Kunkel, B. Kopp, G. Miller, and E. Taub. Constraint-induced movement therapy for motor recovery in choronic stroke patients. Archives of physical medicine and rehabilitation, Vol. 80, (1999), pp.624-828.

[3] P. M. Rossini and F. Pauri. Neuromagnetic integrated methods tracking human brain mechanisms of sensorimotor areas 'plastic' reorganisation. Brain Research Reviews. Vol. 33, (2000), pp.131-154.

[4] S. J. Page, S. Sisto, P. Levine, M. V. Johnston, and M. Hughers. Modified constraint induced therapy: a randomized feasibility and efficacy study. Journal Rehabilitation Research and Development, Vol. 38, (2001), pp.583-590.

[5] S. J. Page, S. Sisto, P. Levine, and R. E. McGrath. Efficacy of modified constraint induced movement therapy in chronic stroke: A single-blinded randomized controlled trial. Archives of Physical Medicine and Rehabilitation, Vol. 85, (2004), pp.14-18.

[6] H. R. Rostami, M. Akbarfahimi, A. M. Hassani, A. R. Akbarinia, and S. Samani. Occupation-based intervention versus rote exercise in modified constraint-induced movement therapy for patients with median and ulnar nerve injuries: A randomized controlled trial. Clinical rehabilitation. Vol.31, (2017), pp.1087-1097.

[7] R. E. Shepherd, and P. D. Gollnick. Oxygen uptake of rats at different work intensities. Pfluegers Archiv, Vol. 362, (1976), pp.219-222.

[8] T. H. Wu, J. J. Lun, W. S. Chen, and F. C. Chong. The electrophysiological and functional effect of shock wave on peripheral nerves. In 29th Annual International Conference of the IEEE. Engineering in Medicine and Biology Society, (2007), pp.2369-2372.

[9] V. Penna, K. Wewetzer, B. Munder, G. B. Stark, and E. M. Lang. The long-term functional recovery of repair of sciatic nerve transection with biogenic conduits. Microsurgery, Vol. 32, (2012), pp.377-382.

[10] E. Taub, G. Uswatte, V. W. Mark, and D. M. Morris. The learned nonuse phenomenon: implications for rehabilitation. Europa Medicophysica, Vol. 42, (2006), pp.241-256. 


\section{Authors}

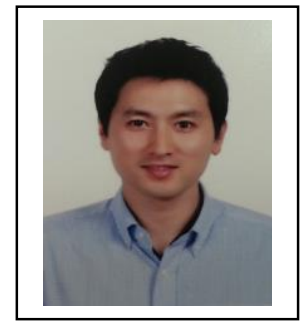

Youn-Bum Sung, he received $\mathrm{PhD}$ degree from Daegu University, Republic of Korea. His research interests include Rehabilitation, Breathing Exercises, Shoulder Disorders, and Sling Exercise. He had completed a lot of training in orthopedic and neurological Sciences Institute. He is currently working as Professor of Department of Physical Therapy, Andong Science College, Republic of Korea.

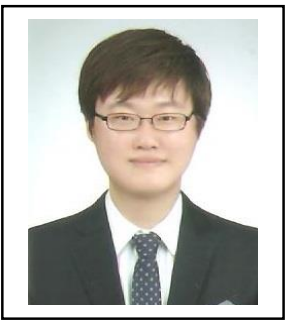

Jung-Ho Lee, he received PhD degree from Daegu University, Republic of Korea. His research interests include Shoulder Disorder, Neurophysiology, Neuroanatomy, Ergonomics, Work Physiology and Sports Physiotherapy. He had completed a lot of training in orthopedic and neurological Sciences Institute. He is currently working as Professor of Department of Physical Therapy, Kyungdong University, Republic of Korea. 